\title{
Seismic fragility analysis with artificial neural networks: Application to nuclear power plant equipment
}

\author{
Zhiyi Wang ${ }^{\mathrm{a}, \mathrm{b}, \mathrm{c}}$, Nicola Pedronic ${ }^{\mathrm{c}}$, Irmela Zentner ${ }^{\mathrm{a}, \mathrm{b}}$, Enrico Zio ${ }^{\mathrm{c}, \mathrm{d}}$ \\ ${ }^{a}$ EDF Lab Saclay, France \\ ${ }^{b}$ Institute for Mechanical Sciences and Industrial Applications, UMR 9219 CNRS-EDF-CEA-ENSTA ParisTech, France \\ ${ }^{c}$ Chair on Systems Science and Energetic Challenge, European Foundation for New Energy of EDF, CentraleSupélec, \\ Université Paris-Saclay, France \\ ${ }^{d}$ Energy Department, Politecnico di Milano, Italy
}

\begin{abstract}
The fragility curve is defined as the conditional probability of failure of a structure, or its critical components, at given values of seismic intensity measures (IMs). The conditional probability of failure is usually computed adopting a log-normal assumption to reduce the computational cost. In this paper, an artificial neural network $(\mathrm{ANN})$ is constructed to improve the computational efficiency for the calculation of structural outputs. The following aspects are addressed in this paper: (a) Implementation of an efficient algorithm to select IMs as inputs of the ANN. The most relevant IMs are selected with a forward selection approach based on semi-partial correlation coefficients; (b) Quantification and investigation of the ANN prediction uncertainty computed with the delta method. It consists of an aleatory component from the simplification of the seismic inputs and an epistemic model uncertainty from the limited size of the training data. The aleatory component is integrated in the computation of fragility curves, whereas the epistemic component provides the confidence intervals; (c) Computation of fragility curves with Monte Carlo method and verification of the validity of the log-normal assumption. This methodology is applied to estimate the probability of failure of an electrical cabinet in a reactor building studied in the framework of the KARISMA benchmark.
\end{abstract}

Keywords: Seismic probabilistic risk assessment; Fragility curve; Artificial neural network; Feature selection; Prediction uncertainty

\section{Introduction}

The seismic probabilistic risk assessment (SPRA) methodology has been applied worldwide for the estimation of the seismic risk of nuclear power plants (NPPs) [1]. In the SPRA methodology, fragility curves are computed as conditional probabilities of failure of structures, or critical components, for given values of a seismic intensity measure (IM), such as the peak ground acceleration (PGA) [2. The core damage frequency of the plant is, then, calculated by the convolution of the fragility curves with the hazard curves in fault tree and event tree analysis [2]. The computation of fragility curves requires a realistic estimation of the structure performance subject to seismic excitations via the quantification and the propagation of uncertainties existing in earthquake ground motions, structural material properties, etc. These uncertainties are categorized into two groups [3]: aleatory uncertainties, which reveal the inherent randomness of variables or stochastic processes, and epistemic uncertainties, which originate from the lack of knowledge about the model and provide a family of confidence interval curves for the fragility estimation. 
In practice, a fragility curve is calculated as the conditional probability that the damage measure (DM) exceeds a critical threshold, for a given seismic IM [4, [5]:

$$
P_{f}(\alpha)=P\left(y>y_{\text {crit }} \mid \alpha\right)
$$

where $y$ is the DM, such as inter-story drift, $y_{\text {crit }}$ is the failure threshold and $\alpha$ represents the seismic IM. This conditional probability can be evaluated pointwise for different $\alpha$ values with the Monte Carlo method [4, 6, as well as with methods based on the log-normal hypothesis [3, 7, 8]. However, both methods require a few hundred heavy numerical simulations with the finite element method (FEM).

One way to improve the computational efficiency consists in building a metamodel to calibrate the statistical relation between seismic inputs and structural outputs. In fact, it is difficult to directly use stochastic ground motions to construct the metamodels, because the high-dimensionality of the inputs of such metamodels requires a very large size of training data to accurately approximate the input-output relation 9 . An alternative is to use seismic IMs as inputs of the metamodels to represent ground motions. Various functional models based on the calibration of IMs-DM relation have been proposed [10, 11, 12]. According to these works, a nonlinear regression metamodel seems more suitable to provide adequate nonlinearity in the IMs-DM relation. However, with this approach, the simplification of the continuous stochastic ground motion by a small set of IMs may not allow to describe all the random variability in the earthquake motion [13. Therefore, it cannot ensure the performance of the metamodels.

Some studies regarding the application of metamodels in fragility analysis have been realized recently. Most works focus on using seismic IMs to characterize earthquake accelerations. Metamodels are constructed to calibrate the relation between DMs and uncertain inputs of the structural models, including IMs and material parameters. The construction of the metamodels is either achieved by decomposing the nonlinear input-output relation with high-dimensional model representation (HDMR) 13, 14, or realized with polynomial regression [15, 16, 17, 18, 19] or other more advanced statistical tools, such as artificial neural networks (ANNs) [20, 21, 22, 23, 24], LASSO regression [25], Bayesian networks [26], merging multivariate adaptive regression splines, radial basis function network, support vector regression [27, Kriging [9, 28], etc. On the other hand, earthquake accelerations are also used directly as inputs of the metamodel in 29. to predict structural response time histories. The construction of the metamodel is divided into two steps: the first step is to extract the characteristics of earthquake motions with nonlinear auto-regression; then the polynomial chaos expansion is applied to these characteristics to construct the metamodel. DMs are computed from the structural response time histories, and fragility curves can be thus obtained. Although this method seems different from the classical metamodeling with IMs, the idea remains the same: the nonlinear auto-regression serves as a tool to extract the features of earthquake motions and past values of the structural displacement, while these features are represented by the IMs in classical approaches. Besides regression methods, classification models like logistic regression, random forests and support vector machine are utilized in [30] to predict directly the probability of failure from the uncertain inputs. Despite the fact that seismic fragility analyses have been successfully performed with different types of metamodels, the following two points are rarely discussed: i) Systematic selection of pertinent IMs to represent seismic ground motions; ii) Quantification of the prediction uncertainty of the metamodels. 
In this paper, a computationally efficient methodology for the application of ANNs to characterize the IMs-DM relation is proposed, from the selection of the most relevant IMs to the quantification of ANN prediction uncertainties. Most existing works take subjective choices of the IMs as inputs of metamodels according to their expertise (e.g. PGA or PGA with other IMs). One IM is obviously not sufficient to represent the seismic ground motion. More systematic approaches are proposed in [20, 23, to guide the selection of IMs. Different sets of IMs are selected to train ANNs in [20] and the performances of the different sets of IMs are analyzed with respect to their corresponding ANNs median training errors. Ferrario et al. proposes a wrapper approach based on genetic algorithms in 23 to select the best subset of IMs. However, these approaches can be time-consuming, because it requires repeated trainings of the metamodel. A more efficient feature selection method is proposed in this work.

The uncertainty in the metamodel predictions is also investigated. The ANN prediction uncertainty is considered to be epistemic in 31 to quantify the impact of the size of the used data. The prediction uncertainty is determined by the bootstrap approach, in which retrainings of ANNs are necessary, and it provides confidence intervals of fragility curves. On the contrary, other works integrate the metamodel uncertainty completely into $P_{f}(\alpha)$ by modeling the standard deviation (Std) of the residual with a dual metamodel (quadratic response surface, HDMR or Kriging) [9, 14, 18, 32. The residual is sampled from a corresponding normal distribution, and it is added to the mean structural DM predicted by the primal metamodel. With this approach, the residual is an aleatory uncertainty, and the influence of the size of the training data is not accounted for. In addition, the number of FEM simulations required by the dual metamodel approach can be very large, because a number of FEM simulations should be performed at every design point with different stochastic motions to obtain the Std. Therefore, it may not be applicable to a very complex structure such as NPP. In this paper, a clearer insight of the ANN prediction uncertainty computed with the delta method is provided: it consists of an aleatory component from the simplification of the seismic inputs and an epistemic uncertainty due to the paucity of the training data. The former is considered in the computation of $P_{f}(\alpha)$, whereas the latter is used in the estimation of confidence intervals.

Among various types of metamodels, ANNs are chosen due to their adequate nonlinearity and their excellent universal approximation capability for continuous bounded functions 33, 34, (e.g. compared to polynomial response surfaces). Firstly, rather than a classification model like a SVM classifier, which returns only binary failed or survived information for the conditions of structures, an ANN regression model provides predictive structural responses and offers more flexibility for the fragility analysis. Furthermore, the applicability of the ANN does not depend on the probability distribution of input data, so it is a versatile model with a very wide domain of application. Finally, a metamodel based on ANN is a regression rather than an interpolation model. If representative seismic IMs are used to characterize the continuous seismic motions as inputs of the metamodel, the IMs cannot fully represent the seismic randomness and this introduces a residual term. However, an interpolation model predicts identical outputs as the original ground motions for the training data: it may thus overfit the input-output relation. This point is addressed in detail in this work.

This paper is organized as follows: in the next section, the basis about simulation-based fragility analysis methods is briefly recalled. Section 3 presents the methodology for ANN-based fragility estimation. Feature 
selection techniques are highlighted in this methodology to select the most relevant seismic IMs for a better accuracy of the metamodels. ANN prediction uncertainties are separated into aleatory and epistemic components. These uncertainties are considered in the computation of the fragility curves and the related confidence intervals. An application of the proposed methodology to the Kashiwazaki-Kariwa NPP is demonstrated in Section 4 in the context of the KARISMA benchmark [35. Conclusions are finally provided in Section 5 . Only the ground motion record-to-record variability is considered in this paper, to better study the impact of the ANN prediction uncertainties on the fragility curves. In addition, without specification, the metamodel mentioned in this paper represents regression or interpolation models, instead of binary classification models.

\section{Simulation-based Fragility Analysis}

A simulation-based fragility analysis is composed of 3 main steps:

1. Structure modeling. This step consists in establishing a set of mathematical partial differential equations to describe the mechanical behavior of the underlying model.

2. Numerical simulation and calculation of the DM. Numerical simulations are performed to propagate the uncertainties and to compute the DM. FEM is the most widely used numerical resolution method.

3. Computation of the conditional probability of failure of the structure. This step is realized by applying a statistical analysis to the IM-DM data cloud $(\alpha, y)$ computed from the numerical simulation results.

In this section, the computation of the DM and the calculation of the conditional probability of failure are further discussed. The concept of the residual of the metamodel is introduced and emphasized. This concept will be later used throughout the next parts of the paper. Two commonly used methods for the computation of the conditional probability are presented. These two methods will be applied to calculate the fragility curves in an industrial complex case study in this paper.

\subsection{Computation of the Damage Measure}

Mechanical model. The mechanical model to compute the DM of a structure or a critical component can be described as

$$
y=f(\boldsymbol{a}(t))
$$

where $\boldsymbol{a}(t)$ represents the seismic ground acceleration. The resolution of Eq. 2 is usually time-consuming, especially when the structural model is very complex. In this way, one needs to resort to the metamodel to reduce the computational cost of the numerical simulations.

Metamodel. In this paper, a metamodel established for IMs-DM relation is desired. It is used to replace the mechanical model in order to improve the computational efficiency:

$$
\hat{y}=\hat{f}\left(\mathrm{IM}_{1}, \mathrm{IM}_{2}, \ldots, \mathrm{IM}_{k}\right)
$$

where the symbol ' $\wedge$ ' denotes the results calculated from the metamodel. The regression of the metamodel leads to a reduction of the variability in the metamodel prediction: $y=\hat{y}+\varepsilon$. The existence of the residual $\varepsilon$ not only comes from the lack-of-fit of the metamodel, but also has more specific interpretations: 
1. The metamodel cannot show sufficient nonlinearity to replace the mechanical model. The residual value can be very high if a linear metamodel is wrongly selected to substitute a nonlinear mechanical model.

2. IMs are adopted to represent the inherent randomness of ground motions $\boldsymbol{a}(t)$, which gives rise to a loss of information in the input variables. Different ground motion time histories with the same set of IM values lead to different structural responses, in contrast to a deterministic response predicted by the metamodel. Consequently, $\varepsilon$ should be present for the training data in the metamodeling process. That is also the main reason why a nonlinear regression model like ANN is preferred, rather than an exact interpolation model, such as Kriging with classical kernels (Gaussian kernel, Matern kernel, etc).

3. The number of the training data for the development of the metamodel is usually limited due to the computational cost of FEM simulations.

These facts show the necessity of the quantification of the metamodel prediction uncertainty, in order to provide reliable applications of metamodels to critical structures such as NPPs.

\subsection{Computation of Fragility Curves}

This section is dedicated to recall the basis of two methods in the computation of fragility curves: the Monte Carlo Method and the regression method. These two methods can be applied to compute the conditional probability of failure when the data cloud $(\alpha, y)$ is provided.

Monte Carlo (MC) method. In this method, $N$ seismic records with the same IM level $\alpha$ are collected. Structural analyses for all $N$ seismic motions are performed, and the probability of failure for the seismic IM level $\alpha$ is calculated as

$$
P_{\mathrm{MC}}(\alpha)=\frac{1}{N} \sum_{i=1}^{N} \mathbf{1}\left[y_{\text {crit }}-y^{i}(\alpha)<0\right]
$$

where $\mathbf{1}\left[y_{\text {crit }}-y^{i}(\alpha)<0\right]$ equals 1 if $y_{\text {crit }}-y^{i}(\alpha)<0$, otherwise it equals 0 .

Regression method with log-normal assumption. The log-normal assumption is commonly adopted to compute the conditional probability of failure. The regression method (Reg), or 'cloud analysis', is based on the linear regression of the data cloud $(\alpha, y)$ in the log-log space [5, 10, 36].

$$
\ln y=c \ln \alpha+\ln b+\varepsilon
$$

where $b$ and $c$ are regression parameters determined from the data cloud $(\ln \alpha, \ln y)$, and the residual $\varepsilon$ follows a normal distribution $\mathcal{N}\left(0, \beta_{R \mid I M}^{2}\right)$. $\beta_{R \mid I M}$ is calculated as

$$
\beta_{R \mid I M}=\sqrt{\frac{\sum_{i=0}^{N}\left(\varepsilon^{i}-\bar{\varepsilon}\right)^{2}}{N-2}}
$$

in which $\bar{\varepsilon}$ is the mean of the regression residuals and $N$ denotes the size of the data $(\alpha, y)$. The conditional probability of failure can be, thus, calculated:

$$
P_{f}(\alpha)=\boldsymbol{\Phi}\left(\frac{\ln b \alpha^{c}-\ln y_{\mathrm{crit}}}{\beta_{R \mid I M}}\right)
$$

where $\boldsymbol{\Phi}(\cdot)$ is the cumulative distribution function of the standard normal distribution $\mathcal{N}(0,1)$.

Both methods MC and Reg are used in the sequel of this paper to compute fragility curves. In particular, the pointwise MC method serves to confirm the validity of the log-normal assumption. 


\section{Description of the ANN-based Seismic Fragility Analysis}

The global procedure for the estimation of the fragility curves with ANNs is illustrated in Figure 1. The basic stages are: (1) Preparation of data set by performing FEM simulations. (2) Feature selection to extract the most important IMs as inputs of the ANN. (3) ANN training and validation. (4) ANN uncertainty quantification. (5) Computation of fragility curves with ANN simulation results.

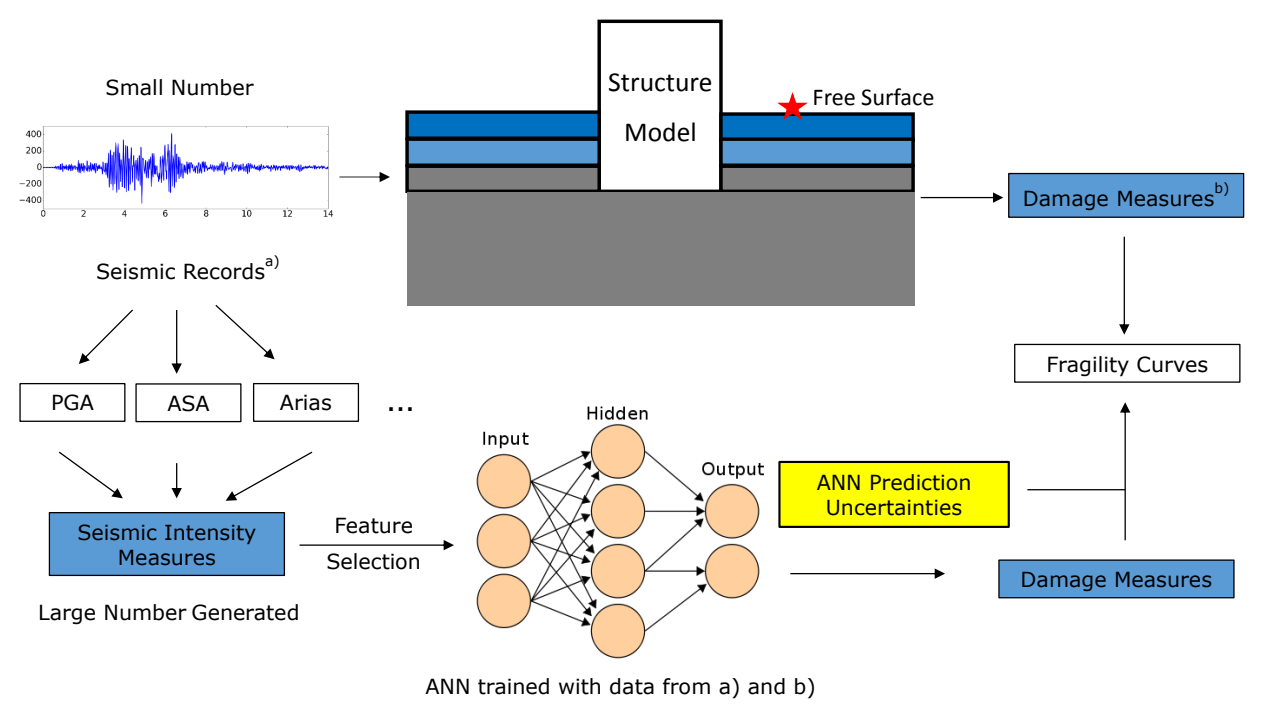

Figure 1: Work flow for the computation of fragility curves with ANN

\subsection{Preparation of Data Set}

This step is achieved by conducting a series of numerical simulations with the FEM. The soil-structure interaction (SSI) should be considered to offer a best estimate of the structural response. The number of simulations is thus limited due to the computational complexity of the FEM analysis, with the presence of the SSI. The basic working flow is divided into the following 5 steps illustrated in Figure 2

1. Generation of synthetic seismic motions at the bedrock. This can be realized by generating a set of seismic motions compatible with the spectral acceleration predicted by the ground motion prediction equations (GMPEs).

2. Convolution of the bedrock accelerations to the free surface. The convolution is performed using a 1D column of soil with the consideration of soil degradation. The degradation of the soil during the earthquake is accounted for by the equivalent linear method (ELM) based on the 1D soil column [37.

3. After the convolution, surface ground motions and their corresponding degraded soil profiles are obtained. The ground motions obtained on the free surface are coherent with the site-specific degraded soil profiles. The latter is utilized as the input of the SSI analysis, whereas IMs of the ground motions on the free surface can be extracted.

4. SSI analysis is conducted and structural response time histories can be thus obtained.

5. The DMs are computed by the post-processing of the structural response time histories.

Consequently, the data set IMs-DM is available for the feature selection and further for the construction of the ANN metamodel. 


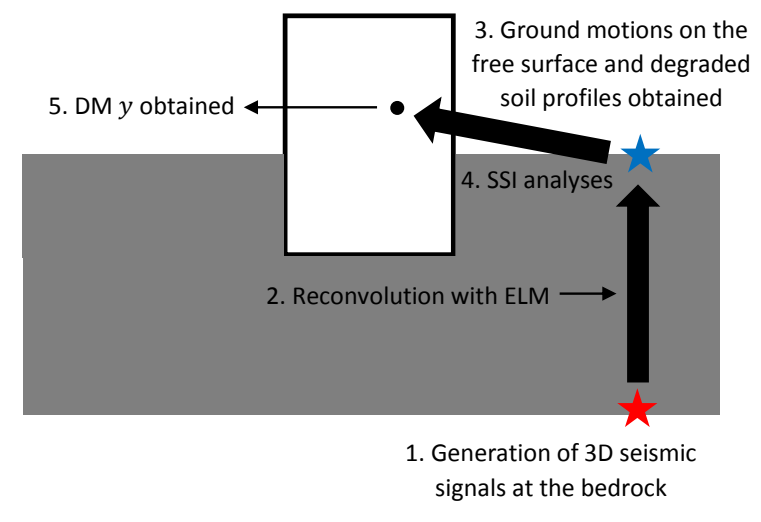

Figure 2: Work flow of FEM simulations

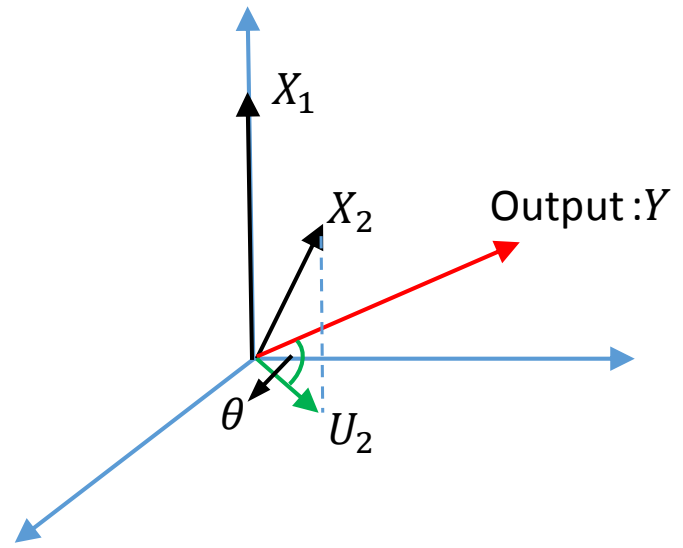

Figure 3: Computation of SPCC $-\cos (\theta)$

\subsection{Feature Selection}

Before the training of the ANNs, it is important to select a subset of IMs whose impact on the DM is dominant. This step, named feature selection, is crucial in the metamodel construction phase to ensure the performance of the ANNs. On the one hand, an IM irrelevant to the output should not be considered as a feature; on the other hand, with the limited size of available training data, a smaller input dimension simplifies the ANN structure and improves the generalization capacities of the network [33]. The feature selection approach applied in 23] is a wrapper approach based on the genetic algorithm (GA). The wrapper approach relies on the metamodel to select the best subset of the features (i.e. relevant IMs) [38. However, one disadvantage of the wrapper approach is its computational burden, because this method requires a large number of repeated trainings of the ANNs.

Filter approach based on semi-partial correlation coefficients. A filter approach is proposed in this paper to improve the computational efficiency of the wrapper feature selection. In machine learning, the filter approach describes a group of methods used to select the features regardless of the metamodel. Therefore, it can be regarded as a sensitivity analysis between inputs and outputs. In the context of fragility analysis, the main difficulty in applying such an approach is the dependence between all the IMs. This correlation should be considered in the execution of the filter approach to discard the redundant information.

For this purpose, a forward selection algorithm driven by semi-partial correlation coefficients (SPCCs) is used to rank the importance of the IMs. As shown in Figure 3 in order to eliminate the dependence, the IM $X_{2}$ is projected onto the orthogonal space of the reference IM $X_{1}$. The SPCC calculates the correlation (the cosine value of the angle $\theta$ ) between the projection $U_{2}$ and $Y$. The orthogonal relation between random variables (RVs) is interpreted by the statistical linear independence between them. Therefore, if IMs follow log-normal distributions, the orthogonal projections can be realized by means of Cholesky factorization on the correlation coefficient matrix of the underlying normally distributed RVs [39. The adopted forward selection algorithm is as follows:

1. Define the input and the output of the algorithm: the input is the feature set $S_{0}=\left\{X_{1}, \cdots, X_{k}\right\}$ $\left(X_{i}=\ln \mathrm{IM}_{i}\right)$ and the output is ranked feature set $S^{*}$. Initialize the output set $S_{0}^{*}=\emptyset$. 
2. Begin the iteration $i$ ( $i$ starts from 0): for each feature $X_{j}$ in $S_{i}$, compute the SPCC between $X_{j}$ and the Output $Y(\ln \mathrm{DM})$, by projecting $X_{j}$ onto the orthogonal space of the ranked feature set $S_{i}^{*}$ (i.e. conditional to $S_{i}^{*}$ ). Select the feature with the largest SPCC value:

$$
\begin{aligned}
j^{*} & =\underset{j}{\arg \max } \operatorname{SPCC}\left(X_{j}, Y \mid S_{i}^{*}\right) \\
R_{i}^{\mathrm{SP}} & =\operatorname{SPCC}\left(X_{j^{*}}, Y \mid S_{i}^{*}\right)
\end{aligned}
$$

When $i=0, \operatorname{SPCC}\left(X_{j}, Y \mid \emptyset\right)$ actually computes the linear correlation coefficient between $X_{j}$ and $Y$.

3. Subtract the selected $X_{j^{*}}$ from the feature set: $S_{i} \ominus X_{j^{*}} \rightarrow S_{i+1}$, and add $X_{j^{*}}$ into the output set: $S_{i}^{*} \oplus X_{j^{*}} \rightarrow S_{i+1}^{*}$.

4. Set $i=i+1$ and return to Step 2 until all the IMs are selected in $S^{*}$.

The IMs are ranked in $S^{*}$ according to their importance to the output, and one can select the first few IMs to train the ANN.

\subsection{ANN Training and Validation}

The structure of a classical, three-layer, feed-forward ANN is illustrated in Figure 4 Mathematically, this ANN consists of activation functions (linear functions, and nonlinear tanh functions) and a set of model parameters. The model parameters are the ANN weights $\boldsymbol{w}$ and biases $\boldsymbol{b}$, which are adjusted by training to minimize a cost function. The cost function computes the difference between the ANN predictions $\hat{y}$ and the targets $y$ (e.g. FEM simulation results), summed over every training example $i$. For simplicity of notation, in this paper, $\boldsymbol{w}$ is used to represent all parameters of the ANNs, including weights and biases.

$$
E(\boldsymbol{x} ; \boldsymbol{w})=\frac{1}{2} \sum_{i=1}^{N}\left(\hat{y}^{i}(\boldsymbol{x} ; \boldsymbol{w})-y^{i}\right)^{2}
$$

where $E(\boldsymbol{x} ; \boldsymbol{w})$ denotes the cost function which the ANN aims to minimize, $N$ is the total number of ANN training examples, and $\boldsymbol{x}$ is the ANN input vector. The ANN is trained based on the gradient vector $\boldsymbol{g}$, which can be computed efficiently by the back-propagation algorithm [33, 40]:

$$
\boldsymbol{g}=\frac{\partial E(\boldsymbol{x} ; \boldsymbol{w})}{\partial \boldsymbol{w}}
$$

For the ANN training, the available data set is divided into 3 independent subsets:

- Training subset (e.g. $60 \%$ of the total data), which is used to determine the optimal weighting parameters $\boldsymbol{w}^{*}$ that minimize the cost function of the ANN model.

- Validation subset (e.g. $20 \%$ of the total data), which supervises the training process. The ANN training is stopped when the validation error reaches its minimum to avoid overfitting [33, 41]. This strategy is called early stopping.

- Test subset (e.g. $20 \%$ of the total data), which is independent of the training and validation subsets. The test subset is not used in the ANN training, but used afterwards to evaluate the generalization capacity of a trained ANN metamodel.

The ANN is suggested to be trained with IMs-DM in log-log space to facilitate the consideration of the ANN uncertainties into the fragility curves. The performance of the ANN can be evaluated by the rootmean-square error (RMSE). Once trained and validated, the ANN substitutes the FEM model to accelerate the computation process. 


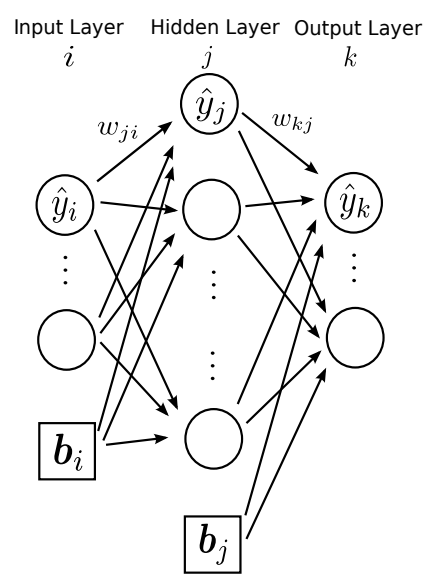

Figure 4: A multiple-layer perceptron model

\subsection{ANN Uncertainty Quantification}

Besides the ANN deterministic prediction $\hat{y}$, the confidence interval (CI) of this prediction can be also estimated. The main methods to evaluate the prediction intervals (PIs) of ANNs are the bootstrap method, the Bayesian approach and the delta method [42. The delta method is adopted in this study due to its computational efficiency because it does not require repeated trainings of the ANNs with the bootstrap resampling 43. Assuming a normal distribution of the ANN training error, this method relies on the linear Taylor expansion of the ANN model and estimates the PIs of the corresponding linear model [43, 44, 45]. In this way, the Hessian matrix of the ANN is approximated by the product of the Jacobian matrices. Mathematically, the PIs are computed with the Std of the ANN training error $\sigma_{\mathrm{ANN}}$ and the gradient vector $h$ :

$$
\boldsymbol{h}^{i}=\frac{\partial \hat{y}^{i}}{\partial \boldsymbol{w}}=\frac{\partial \hat{y}^{i}}{\partial E(\boldsymbol{x} ; \boldsymbol{w})} \frac{\partial E(\boldsymbol{x} ; \boldsymbol{w})}{\partial \boldsymbol{w}}=\frac{\boldsymbol{g}}{\hat{y}^{i}-y^{i}}
$$

The Jacobian matrix $\boldsymbol{J}$ of the ANN training data is, hence, constructed as

$$
\boldsymbol{J}=\left[\begin{array}{llllll}
\boldsymbol{h}^{1} & \boldsymbol{h}^{2} & \cdots & \boldsymbol{h}^{i} & \cdots & \boldsymbol{h}^{N}
\end{array}\right]
$$

where $\boldsymbol{J}$ is a $Q \times N$ matrix, with $N$ the number of the ANN training examples and $Q$ the number of the weighting parameters in the ANN. Consequently, the prediction uncertainties of ANNs are calculated as

$$
s^{2}=\sigma_{\text {ANN }}^{2}+\sigma_{\text {ANN }}^{2} \boldsymbol{h}_{\text {test }}^{T}\left(\boldsymbol{J} \boldsymbol{J}^{T}\right)^{-1} \boldsymbol{h}_{\text {test }}
$$

where $s$ denotes the Std of the ANN predictions.

The source of the ANN prediction uncertainty comes from two aspects: i) The selected IMs cannot completely represent the variability of the ground motion. This eventually reduces the variability of the output. ii) The ANN accuracy due to the limited size of data to train ANNs. It is the statistical uncertainty linked to the ANN model. Let us return to the two components in $s^{2}$ : the first fixed part $\sigma_{\text {ANN }}^{2}$ represents the ANN training error, and the second part $\sigma_{\mathrm{ANN} \text {,stat }}^{2} \triangleq \sigma_{\mathrm{ANN}}^{2} \boldsymbol{h}_{\text {test }}^{T}\left(\boldsymbol{J}^{T}\right)^{-1} \boldsymbol{h}_{\text {test }}$ depends on the training and the test data.

1. The first term $\sigma_{\mathrm{ANN}}^{2}$ estimates the difference between the FEM simulation results and the predictions of the ANN. Given that a nonlinear regression returns a regular hyper-surface in a high dimensional space, 
the predictions of the ANN show always less variability than the original FEM data. As discussed in Section 2.1. this phenomenon is mainly due to the loss of the inherent seismic randomness in the input variables, so that the nature of $\sigma_{\mathrm{ANN}}^{2}$ can be regarded as the aleatory uncertainty not explained by the ANN input parameters.

2. The second term $\sigma_{\text {ANN,stat }}^{2}$ is the statistical uncertainty linked to the limited data used to train and test the ANNs. The information of the training data is included in the $\boldsymbol{J}$ matrix and $\boldsymbol{h}_{\text {test }}$ incorporates the influence of the test data. It is thus considered as the epistemic uncertainty, and it provides the confidence intervals of the fragility curves.

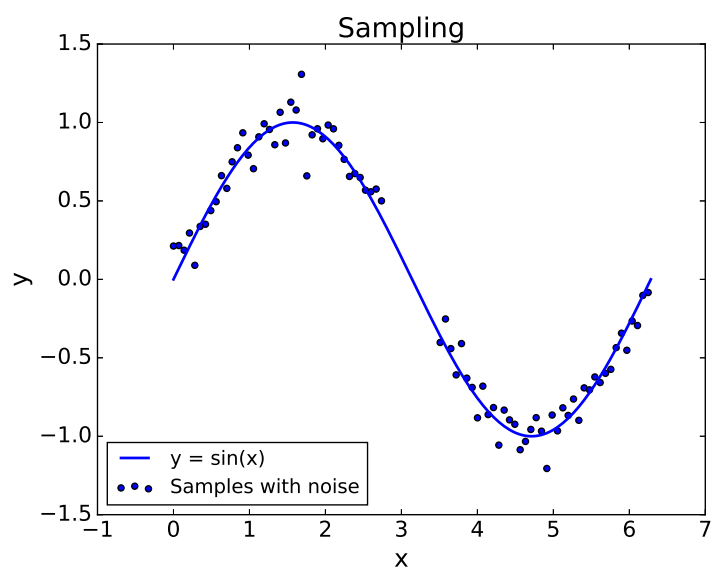

(a) ANN Training data

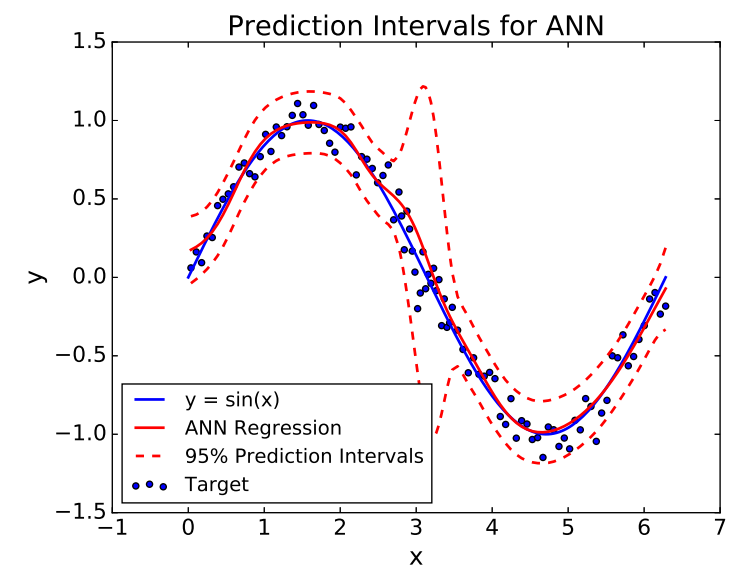

(b) ANN Test results

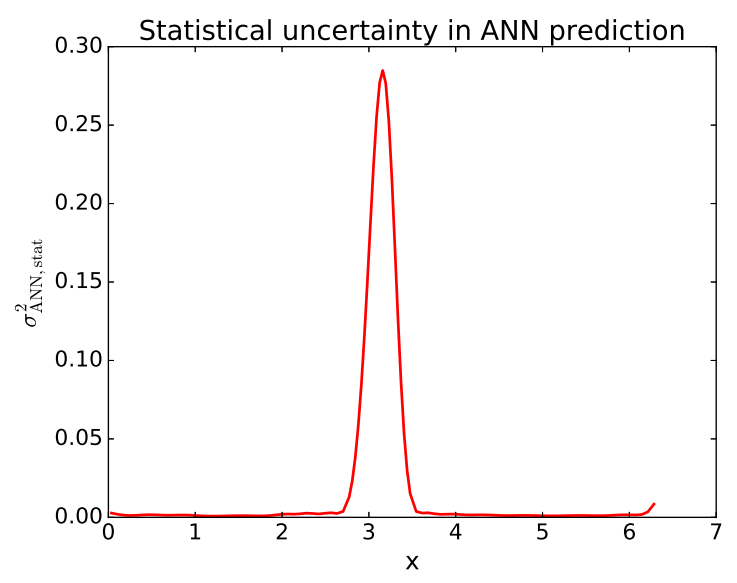

(c) ANN statistical uncertainty

Figure 5: An illustrative example for ANN prediction uncertainties: (a) 80 sparse training data $y=\sin (x)+z$, with $z \sim$ $\mathcal{N}\left(0,0.1^{2}\right)$. An ANN is trained with $(x, y) . z$ is assumed to be the unidentified input. No training data are generated near $x=3$, for the purpose of checking the property of $\sigma_{\mathrm{ANN} \text {,stat }}$ (b) ANN is trained, with $\sigma_{\mathrm{ANN}}=0.0931$, close to the Std of $z$. The ANN is then tested on 100 uniformly regenerated data. (c) $\sigma_{\mathrm{ANN}}$,stat with a peak near $x=3$, where no training data exist. It can be concluded that $\sigma_{\mathrm{ANN} \text {,stat }}$ captures the scarcity of the training data.

These two aspects are illustrated with a simple case study in Figure 5. In fact, $\sigma_{\mathrm{ANN}}$ should have contained also an epistemic uncertainty contribution from the insufficient nonlinearity of the ANN. However, the high flexibility of the ANN architecture offers an universal approximation capacity to continuous bounded functions 34. If the number of the hidden layer units is correctly determined, the error from the ANN nonlinearity 
can be considered less important compared to the aleatory randomness neglected in its inputs. This can be observed from Figure 5 in spite of the existence of the unidentified input $z$, the ANN regression curve stays very close to $y=\sin (x)$ curve in the training data region. Consequently, this epistemic contribution is assumed negligible in this study. Next section presents the computation of fragility curves with the consideration of these two uncertainties.

\subsection{Fragility Curves with ANN simulation results}

The marginal distributions of the IMs are known through the GMPEs [46. Correlation models are also available in the literature [47. Based on GMPEs and correlation models, seismic IMs can be generated directly as inputs of the ANN. One advantage of using IMs as inputs of metamodels is that no seismic ground motions are in need for ANN simulations.

Given the large number of simulation results provided by ANN, fragility curves can be computed with methods MC and Reg. This also allows confirming the validity of the log-normal assumption used in the fragility analysis. It is important to take into account the ANN prediction uncertainty, because the regression of the ANN reduces the statistical variability of the DMs, and thus the aleatory uncertainty of the fragility curves. As a consequence, methods MC and Reg should be adapted with the consideration of the ANN prediction uncertainty.

For the Reg method, as the ANN is trained in log-log space, the aleatory residual uncertainty $\sigma_{\text {ANN }}^{2}$ can be integrated directly into the expression of the log-normal CDF (Eq. 7). The basic steps consist of:

1. Generation of IMs as inputs of the ANN. Select one IM $\alpha$ as the parameter of the fragility curve.

2. Conduct ANN simulations with the generated IMs. The ANN outputs $\hat{y}$ are obtained.

3. Apply Reg method on data $(\alpha, \hat{y})$ and determine the regression parameters $b, c, \beta_{R \mid I M}^{\mathrm{ANN}}$.

4. Compute the conditional probability of failure with the consideration of $\sigma_{\mathrm{ANN}}^{2}$ :

$$
P_{f}(\alpha)=\boldsymbol{\Phi}\left(\frac{\ln b \alpha^{c}-\ln y_{\text {crit }}}{\beta_{\text {Total }}}\right)=\boldsymbol{\Phi}\left(\frac{\ln b \alpha^{c}-\ln y_{\text {crit }}}{\sqrt{\left(\beta_{R \mid I M}^{\mathrm{ANN}}\right)^{2}+\sigma_{\mathrm{ANN}}^{2}}}\right)
$$

A similar idea concerning the consideration of this metamodel uncertainty has been used in [28] for Kriging.

For the MC estimation based on the ANN simulation data, no analytical expression can be derived. One has to resort to the sampling of the residual, for both aleatory and epistemic parts. The basic steps are:

1. Generation of $N$ groups of seismic IMs as inputs of the ANN, conditional to the IM $\alpha$ used as the independent parameter of the fragility curve.

2. Conduct ANN simulations with the generated IMs. The ANN outputs $\hat{y}$ are obtained.

3. Sampling of $N$ residuals $\varepsilon_{\mathrm{ANN}}$ following $\mathcal{N}\left(0, \sigma_{\mathrm{ANN}}^{2}\right)$, and sampling of $\varepsilon_{\mathrm{ANN}, \mathrm{stat}}^{\gamma}$, the $\gamma$ percentile of $\mathcal{N}\left(0, \sigma_{\text {ANN,stat }}^{2}\right)$.

4. Computation of the conditional probability of failure $P_{\mathrm{MC}}(\alpha)$ and the $\gamma$ confidence interval $\tilde{P}_{\mathrm{MC}}^{\gamma}(\alpha)$ :

$$
\begin{aligned}
& P_{\mathrm{MC}}(\alpha)=\frac{1}{N} \sum_{i=1}^{N} \mathbf{1}\left[\ln y_{\mathrm{crit}}<\ln \hat{y}^{i}(\alpha)+\varepsilon_{\mathrm{ANN}}^{i}\right] \\
& \tilde{P}_{\mathrm{MC}}^{\gamma}(\alpha)=\frac{1}{N} \sum_{i=1}^{N} \mathbf{1}\left[\ln y_{\mathrm{crit}}<\ln \hat{y}^{i}(\alpha)+\varepsilon_{\mathrm{ANN}}^{i}+\varepsilon_{\mathrm{ANN}, \mathrm{stat}}^{\gamma}\right]
\end{aligned}
$$


5. Repeat the steps 1-4 for different $\alpha$ values.

$P_{\mathrm{MC}}(\alpha)$ can be compared with $P_{f}(\alpha)$ to confirm the assumption of log-normality of the fragility curve. For the purpose of simplicity, the adapted Reg and MC methods in the ANN-based fragility analysis are denoted as 'Modified Reg' and 'Modified MC', respectively. In the sequel, the described methodology is illustrated with an industrial test case studied in the KARISMA benchmark.

\section{Case Study: KARISMA benchmark}

\subsection{Kashiwazaki-Kariwa FEM Analysis}

In 2007, the Japanese Kashiwazaki-Kariwa (K-K) NPP was affected by the Niigataken-Chuetsu-Oki earthquake (NCOE) with a magnitude $M_{w}=6.6$ and an epicenter distance of $16 \mathrm{~km}$. The structure of the K-K NPP is shown in Figure 6. In this paper, we are interested in the reliability of a hypothetical electrical cabinet located on the fifth floor of the Unit 7 reactor building of the NPP (Figure 6). The finite element model for the Unit 7 consists of 92,000 degrees of freedom with 10,700 nodes and 15,600 elements, including bar, beam, and different shell elements. The constitutive law of the materials is considered as linear. The NPP model is embedded 23 meters in the soil, which is accounted for in the SSI analysis. The structural analyses are carried out with Code_Aster, a finite element analysis open-source software developed by EDF group [48, while the soil part is solved with MISS based on the boundary element method (BEM) [49.

FEM analyses are performed according to the approach described in Section 3.1. 100 triplets of 3D synthetic ground motions are generated at the bedrock with $V s_{30}=720 \mathrm{~m} / \mathrm{s}$ and used for the uncertainty propagation. Given the NCOE scenario, the generation of the synthetic ground motions are based on scenario spectra predicted by the Campbell-Bozorgnia 2008 (C\&B 2008) GMPE [46]. In order to obtain sufficient failure cases for the fragility analysis, the synthetic seismic motions at the bedrock are scaled with a factor of three. After analyses with ELM, 100 triplets of ground motions on the free surface and 100 degraded soil profiles are obtained.

The impedances of the soil and the seismic forces should have been computed for each soil profile using BEM. However, the high complexity of the embedded foundation makes it hard to achieve: it takes 24 hours to run the BEM simulation for one soil profile. In order to reduce the computational cost, the 3D seismic signals at the bedrock are regrouped into four soil classes according to their PGA values: i. PGA $\in[0,0.5 \mathrm{~g})$ ii. $\mathrm{PGA} \in[0.5 \mathrm{~g}, 1.0 \mathrm{~g})$ iii. $\mathrm{PGA} \in[1.0 \mathrm{~g}, 1.5 \mathrm{~g})$ iv. $\mathrm{PGA} \in[1.5 \mathrm{~g},+\infty)$. The degraded soil profiles are averaged within each class and four soil profiles are obtained to represent four different degradation levels. The SSI analyses are performed with the 100 ground motions on the free surface, as well as the impedances and seismic forces calculated from the four soil profiles, to compute the floor accelerations of the K-K NPP.

Anchorage failure of the electrical cabinet is considered in this study. The capacity is given by the floor spectral acceleration of the anchorage point around $4 \mathrm{~Hz}$, the assumed natural frequency of the cabinet. The maximum value of the floor spectral accelerations in the two horizontal directions, integrated over a frequency interval around $4 \mathrm{~Hz}$ to account for the uncertainty, is defined as the DM $y$ :

$$
y=\max _{i=X, Y} \int_{3.5}^{4.5} S_{a, i}^{e}(f) \mathrm{d} f
$$


where $S_{a, i}^{e}$ denotes the spectral acceleration of the electrical equipment in the $i$-th direction. Figure 7 shows the 100 calculated DMs as a function of the geometric mean of the PGAs of the horizontal seismic motions on the free surface.

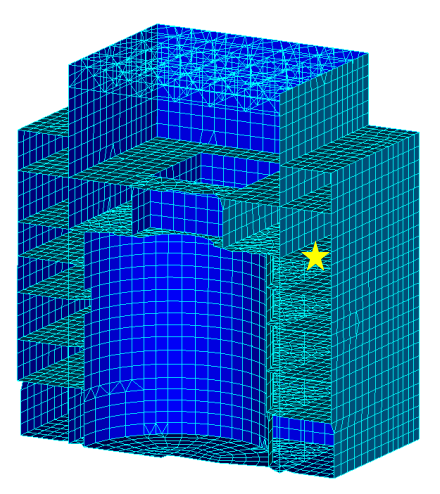

Figure 6: Location of the electrical cabinet in the K-K model (indicated by the star symbol)

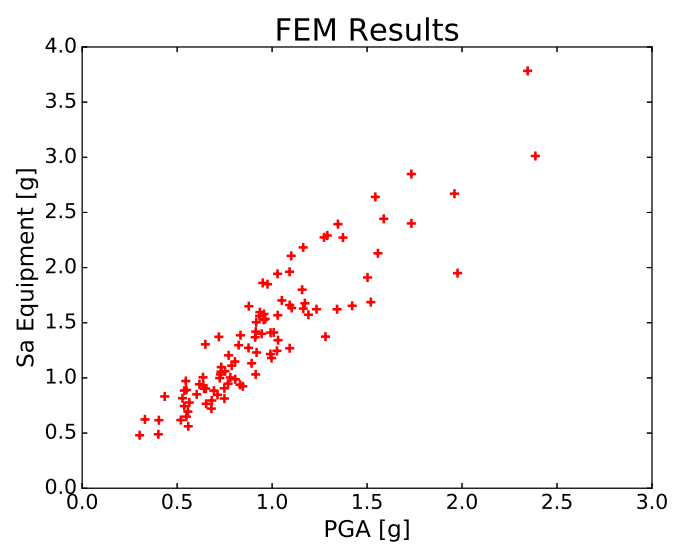

Figure 7: Point cloud of DMs (Eq. 18 calculated with

FEM

\subsection{Data Analysis}

The 100 IMs-DM obtained from FEM simulations can be used for the construction and the training of the ANN metamodel. 8 classical IMs are chosen as candidates for the inputs of the ANN metamodel. The 8 IMs include the commonly used seismic intensity indicators PGA, PGV, PGD, $\mathrm{PS}_{a}\left(f_{0}\right), \mathrm{CAV}$, Arias intensity $I_{A}$, as well as the predominant period $T_{p}$ used in [50] and the ASA proposed in [51]. These IMs are presented in detail in Table 1. The geometric means of IMs in the two horizontal directions are used as scalar IMs for 3D ground motions. The integration domain of the ASA is slightly modified compared to its initial definition in [51, to consider the uncertainty on the natural frequency of the electrical cabinet.

Table 1: Definitions of classical seismic intensity measures

\begin{tabular}{ccc}
\hline Intensity Measures & Definitions & Comments \\
\hline PGA (peak ground acceleration) & $\max |a(t)|$ & $a(t):$ seismic acceleration \\
PGV (peak ground velocity) & $\max |v(t)|$ & $v(t):$ seismic velocity \\
PGD (peak ground displacement) & $\max |u(t)|$ & $u(t):$ seismic displacement \\
$\mathrm{PS}_{a}\left(f_{0}\right)$ (pseudo-spectral acceleration) & Spectral acceleration & $f_{0}=4 \mathrm{~Hz}$, damping $5 \%$ \\
$\mathrm{ASA}$ (average spectral acceleration) & $\int_{3.5}^{4.5} \mathrm{PS}_{a}(f) \mathrm{d} f$ & $f:$ frequency \\
$T_{p}$ (predominant period) & $\arg \max _{T} \mathrm{PS}_{a}\left(\frac{1}{T}\right)$ & $T=1 / f$ \\
$\mathrm{CAV}$ (cumulative absolute velocity) & $\int_{0}^{t_{\max }}|a(t)| \mathrm{d} t$ & $t_{\text {max }}$ : total seismic duration \\
$I_{A}$ (Arias intensity) & $\frac{\pi}{2 g} \int_{0}^{t_{\max }} a(t)^{2} \mathrm{~d} t$ & $g=9.81 \mathrm{~m} / \mathrm{s}^{2}$ \\
\hline
\end{tabular}

The correlation coefficients $\rho$ between the eight IMs and the DM defined by 18 are listed in Table 2 It can be observed that, among all the eight chosen IMs, ASA is the most relevant IM to the DM, whereas there is a very weak correlation for $T_{p}$.

Table 2: Correlation coefficients between IMs and DM

\begin{tabular}{ccccccccc}
\hline Intensity Measures & PGA & PGV & PGD & PS $_{a}$ & ASA & $T_{p}$ & CAV & $I_{A}$ \\
\hline$\rho$ & 0.913 & 0.693 & 0.420 & 0.920 & 0.950 & 0.093 & 0.889 & 0.890 \\
\hline
\end{tabular}


The statistical distributions of the eight selected IMs are examined to check their log-normality. The eight proposed IMs are normalized and compared to $\mathcal{N}(0,1)$. The normalization is realized by

$$
\alpha_{\mathrm{Norm}}=\frac{\ln \alpha-\mu_{\ln \alpha}}{\sigma_{\ln \alpha}}
$$

where $\mu_{\ln \alpha}$ and $\sigma_{\ln \alpha}$ denote the mean and the $\operatorname{Std}$ of $\ln \alpha$, respectively. For simplicity of illustration, the probability plots of three IMs (PGA, ASA and $I_{A}$ ) are shown in Figure 8. Besides, the values of the coefficients of determination $R^{2}$ of the probability plots are given in Table 3 , for all the eight IMs: the closer to the log-normal distribution the IM is, the closer to 1 the value of $R^{2}$ will be. It can be concluded that it is reasonable to apply the log-normal distribution model to all eight IMs.

This verification is performed because: i) For the selection of the subset of IMs in Section 3.2 the Cholesky factorization is executed on the covariance matrix of Gaussian random variables (RVs). As a result, if the IMs follow log-normal distributions, the Cholesky factorization can be directly applied to $\ln (\mathrm{IMs})$. ii) For the generation of IMs in the ANN simulation part in Section 3.5. one needs to know the marginal distribution of the IMs to be generated. In this way, it can be confirmed that the marginal distributions of the IMs are effectively log-normal. If the IMs are not log-normally distributed, an additional Nataf transformation [39] should be carried out, to transform arbitrary RVs to Gaussian RVs, in both Section 3.2 and Section 3.5 .

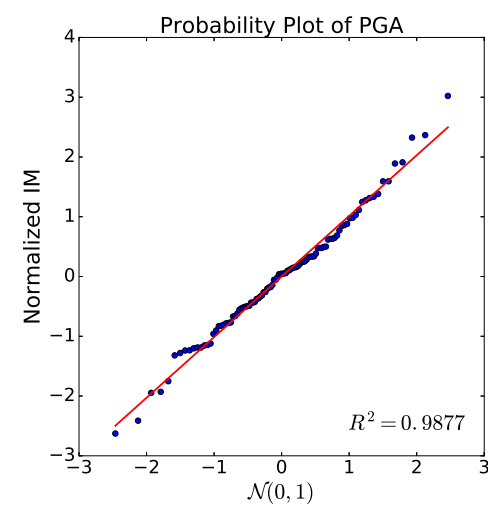

(a) Probability plot for PGA

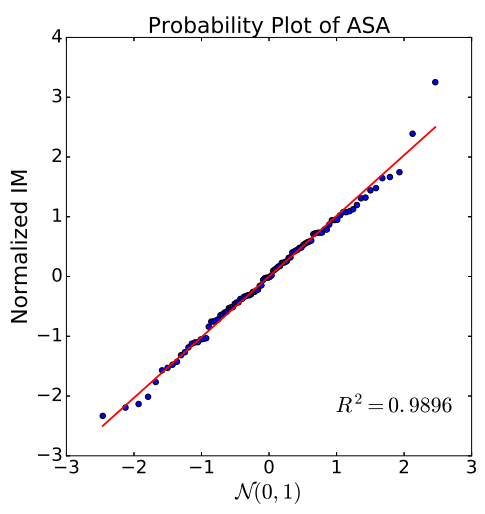

(b) Probability plot for ASA

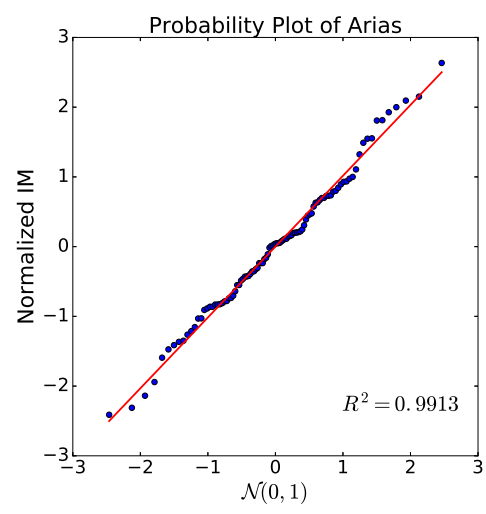

(c) Probability plot for $I_{A}$

Figure 8: Probability plots for PGA, ASA and $I_{A}$ to check their log-normality

Table 3: Coefficients of determination of the probability plots

\begin{tabular}{ccccccccc}
\hline Intensity Measures & PGA & PGV & PGD & PS $_{a}$ & ASA & $T_{p}$ & CAV & $I_{A}$ \\
\hline$R^{2}$ & 0.9877 & 0.9970 & 0.9915 & 0.9866 & 0.9896 & 0.9823 & 0.9912 & 0.9913 \\
\hline
\end{tabular}

\subsection{Selection of Relevant IMs and Determination of ANN Structures}

Feature selection. Due to the limited size of the data set (100 IMs-DM), it is necessary to apply feature selection to obtain a reliable ANN metamodel with good generalization capabilities. A very complex ANN with a large number of unknown weights can easily to be overfitted, given the underlying data set. The features (IMs) are selected with the SPCC filter approach proposed in Section 3.2. The result after the feature selection with SPCC is illustrated in Figure 9. From the forward selection result, ASA and $I_{A}$ are 
selected as the relevant features because the $R^{\mathrm{SP}}$ for the other IMs are less than 0.05 , so that they can be regarded as non-influential if ASA and $I_{A}$ have already been considered.

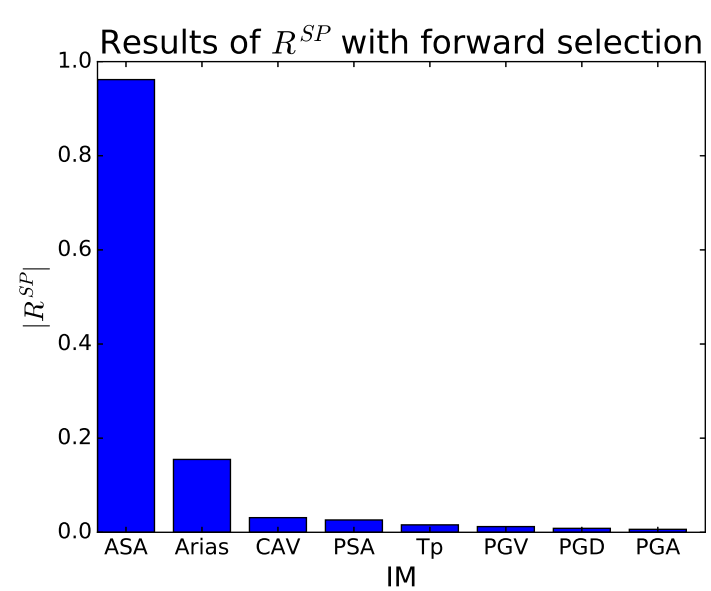

Figure 9: Results of forward selection

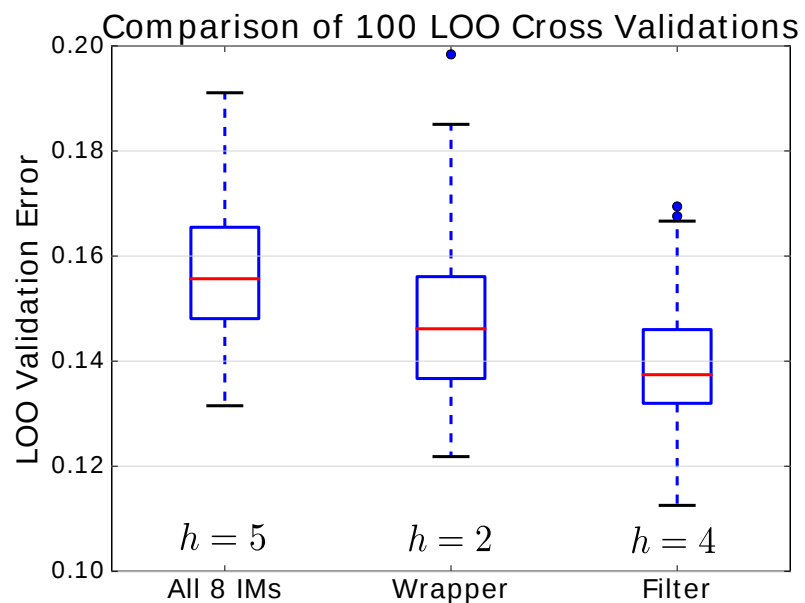

Figure 10: LOO cross-validation results for different ANNs

The number of the neurons $h$ in the hidden layer should be determined additionally, because the filter approach only selects the most relevant feature subset. For this purpose, the total set of 100 data is separated into 2 subsets: 80 data for training and cross-validation (T-CV data), and 20 data for test. The 20 test data are not used to determine the structure of the ANN, but used later in Section 4.4 to evaluate the generalization capacity of the trained ANN. 4-fold cross-validation based on $80 \mathrm{~T}-\mathrm{CV}$ data is executed on ANNs with $h=1,2, \ldots 8$ of hidden neurons, respectively, in order to select the optimal number: The ANN structure with the smallest 4-fold cross-validation error is chosen for the final metamodel. The optimal value of $h$ determined by the described approach is 4 .

Comparison of selected features. We compare our results to the features selected with the wrapper approach based on GA used in [23]. The final feature subset selected by GA is ASA and CAV, with $h=2$ in the hidden layer. It can be observed that both approaches select similar seismic IMs: ASA, the most efficient IM in this study (Table 2), and an IM concerning the integration of the ground motion acceleration over the signal duration $\left(I_{A}\right.$ or $\left.\mathrm{CAV}\right)$.

In order to highlight the necessity of the feature selection, the leaving-one-out (LOO) cross-validation is carried out with the ANN structures determined by the feature selection procedures, as well as the ANN without dimensionality reduction (i.e. with all eight IMs. The number of hidden nodes is $h=5$ ). With random initializations of ANN weighting parameters, 100 LOO cross-validations have been performed. The box-plot of the LOO cross-validation error is shown in Figure 10. From the results, one can observe that the ANN models determined by feature selections show more accuracy. In addition, the ANN model with the filter approach performs best in the LOO cross-validation.

Therefore, in the sequel of this paper, the ANN is trained with two inputs (ASA and $I_{A}$ ), four hidden layer nodes and one output, which is computed according to Eq. 18. The trained ANN is used for all runs of ANN simulations. Point clouds and fragility curves will be plotted with ASA, which is the most efficient IM in this study. 


\subsection{Results of the ANN Training}

Training results. Training based on the back-propagation algorithm is carried out with the ANN structure determined by the filter approach. The ANN toolbox used in this study is an open-source python package 'Neurolab' with the self-implemented delta method for the quantification of ANN prediction uncertainties. The 80 T-CV data in Section 4.3 is again divided into 2 subsets: 60 data for training and 20 data for validation. Early stopping is applied on the validation set to avoid overfitting. The generalization capacity of the ANN is examined on the 20 test data. The ANN is trained in log-log space. The results of the ANN training, as well as the point clouds of the ANN outputs $\hat{y}$ of the test data are shown in Figure 11 and Figure 12. From Figure 11, one can conclude that the training results are satisfactory. Most of the results in the 'prediction-target' space are located in the neighborhood of the dashed diagonal line. The ANN prediction results for the test data set in Figure 12 reveal a globally satisfactory prediction quality: the ANN predictions remain coherent with the FEM results. In fact, with a regression model like ANN, it is not possible to obtain the exact prediction results. In addition, it has to be pointed out that the dispersion of the ANN predictions is reduced compared to the FEM results. This is due to the loss of the aleatory uncertainty by reducing ground motions to two IMs in the ANN metamodeling. The underestimated variability in the ANN predictions will reduce the uncertainty in the fragility curve. The histogram of the normalized ANN training residuals is plotted in Figure 13. It can be observed that its distribution is close to $\mathcal{N}(0,1)$, so that the assumption of normality of the ANN residuals in the delta method can be considered reasonable in this study.

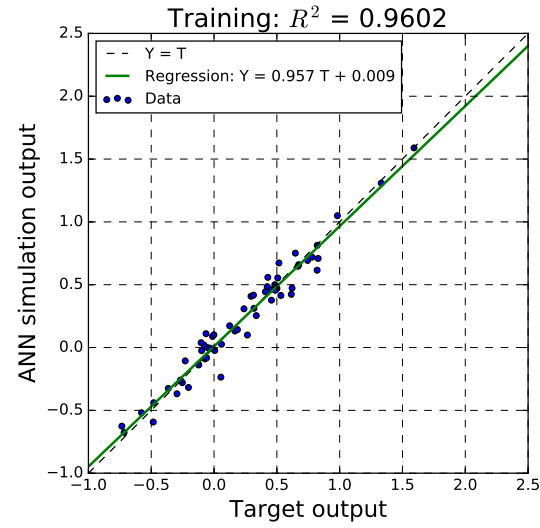

(a) Training set

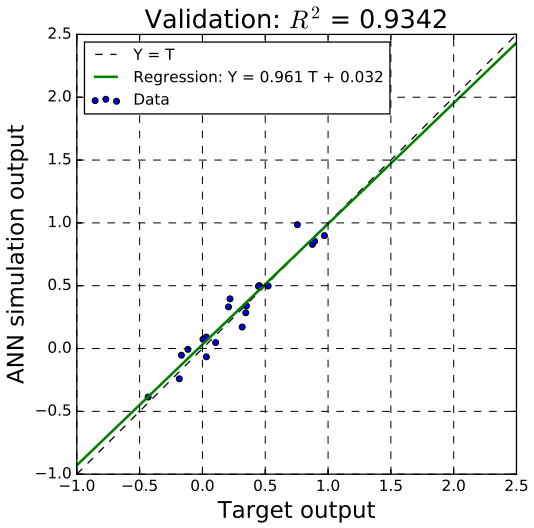

(b) Validation set

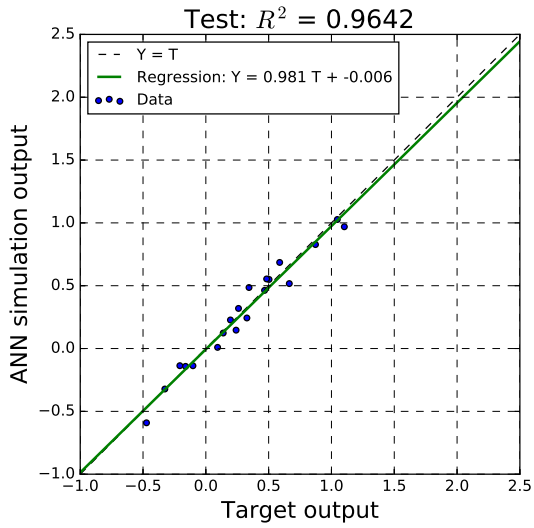

(c) Test set

Figure 11: ANN training results

Comparison with other metamodels. The training results of the ANN are compared with those of other metamodels, including Kriging with Gaussian kernel (an interpolation model), Kriging with Gaussian and White noise kernel (a regression model) and quadratic response surface. The metamodels are constructed with $80 \mathrm{~T}-\mathrm{CV}$ data and tested on 20 test data, using the python toolbox scikit-learn. The RMSE between metamodel predictions and FEM outputs is used to evaluate the accuracy of the different metamodels. The seismic IMs used are ASA and $I_{A}$, the same used for the ANN. The results are reported in Table 4.

Several conclusions can be drawn from Table 4 i) Kriging interpolation is not an appropriate metamodel for this study, since the test error is much larger than other models. The reason has already been discussed 


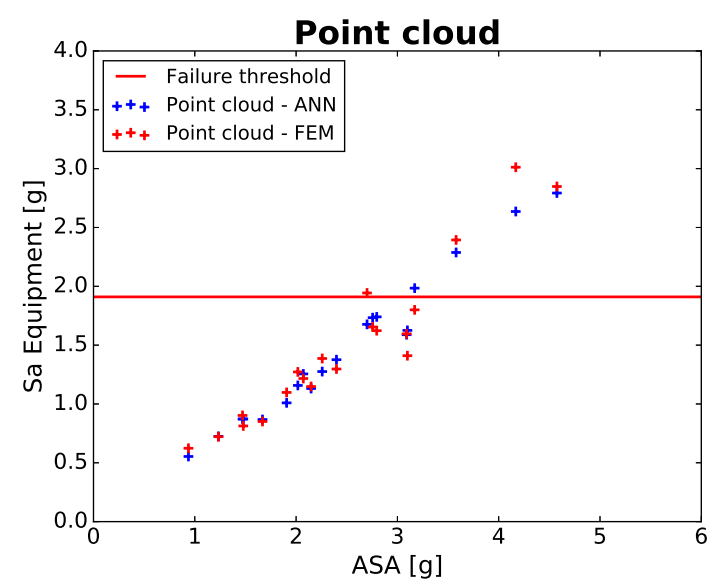

Figure 12: ANN test point cloud

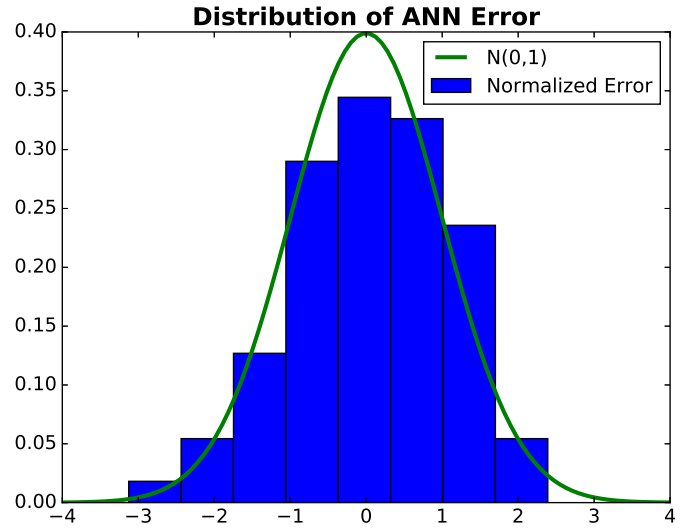

Figure 13: Distribution of ANN training residuals

Table 4: Training and test results for different metamodels

\begin{tabular}{ccc}
\hline Model & RMSE Training (80 data) & RMSE Test (20 data) \\
\hline ANN & 0.141 & 0.135 \\
Kriging interpolation (Gaussian kernel) & 0 & 0.43 \\
Kriging regression (Gaussian+White noise kernel) & 0.153 & 0.145 \\
Quadratic response surface & 0.151 & 0.151 \\
\hline
\end{tabular}

in Section 2.1 the zero residual in the training of Kriging overfits the model. The generalization capability of the interpolation Kriging model is thus very limited with the underlying data. ii) Once the residual is present in the training data of the Kriging regression, the performance of the Kriging is largely improved. iii) Quadratic response surface offers less nonlinearity than ANN, which is why its errors are larger. iv) Overall, ANN shows slightly better performance than other considered metamodels.

Consideration of ANN prediction uncertainties. The ANN model is validated in the previous subsections. Let us show in this part the necessity of the incorporation of the $\sigma_{\mathrm{ANN}}$ in the fragility curves. We focus on the $80 \mathrm{~T}-\mathrm{CV}$ data used to train the ANN. Based on the same 80 seismic inputs, one can obtain 80 structural outputs $y$ and $\hat{y}$, from FEM and ANN simulations respectively. Fragility curves are computed with Reg method (Eq. 7) for data set $(\alpha, y)$ and $(\alpha, \hat{y})$. MC method could have also been used for FEM results. However, the high complexity of the K-K model makes it very difficult to perform adequate FEM simulations for the MC estimation. This is also one of the main motivations to construct a metamodel in this study: the metamodel provides the possibility to conduct a non-parametric fragility analysis. The fragility curves are calculated for $\alpha=\mathrm{ASA}$ and $\alpha=I_{A}$, respectively, in order to provide further discussions. At the same time, the 'modified Reg' method (Eq. 15) is applied to the data set $(\alpha, \hat{y})$ to compute fragility curves, accounting for the aleatory residual uncertainty of the ANN predictions. From Figure 14, it can be seen that:

- A clear difference between the fragility curves computed with FEM Reg and ANN Reg can be observed. The difference is much less evident for $I_{A}$ than ASA. Nevertheless, this is not due to the fact that the ANN metamodel is poorly calibrated, because the ANN accuracy has been validated above and it is even better than other possible metamodels. If the fragility curves calculated with Reg method are not coherent for the training data between FEM and ANN, one can hardly trust the conditional probability of failure computed 


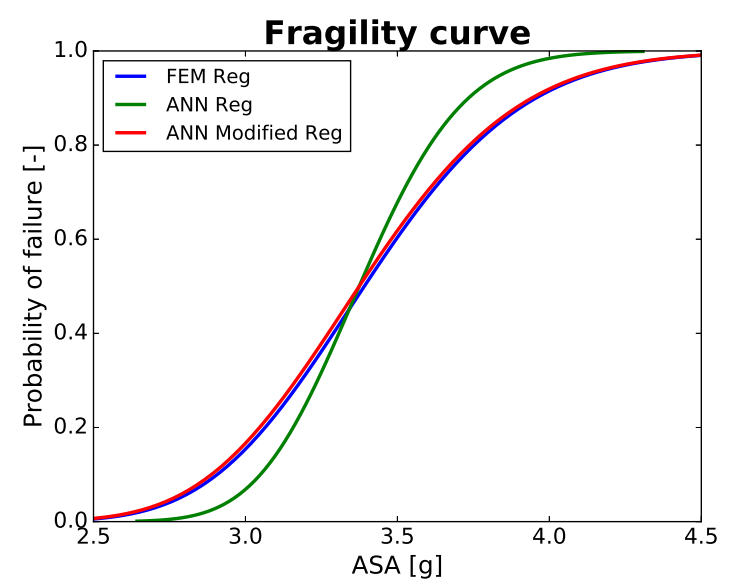

(a) Fragility curves computed with 80 T-CV data as function of ASA

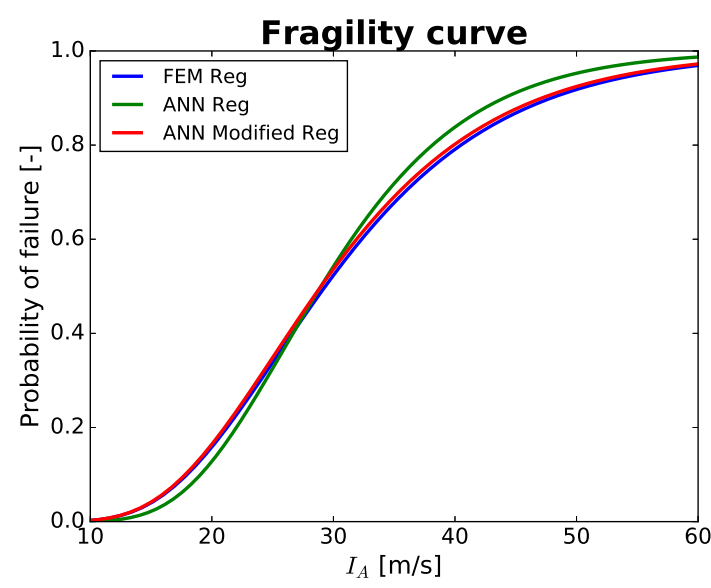

(b) Fragility curves computed with 80 T-CV data as function of $I_{A}$

Figure 14: Fragility curves computed with 80 T-CV data

with the ANN based on other test data.

- The difference is due to the aleatory part of the ANN prediction uncertainty $\sigma_{\mathrm{ANN}}$. It represents the seismic inherent randomness not identified in the inputs (ASA and $I_{A}$ ) of the ANN metamodel. Once $\sigma_{\text {ANN }}$ is integrated in the computation of the conditional probability with Eq. 15, the computed fragility curves almost coincide with the FEM Reg curves, for both ASA and $I_{A}$.

- $\sigma_{\mathrm{ANN}}$ has less influence when the fragility curves are plotted for $I_{A}$. It is because $I_{A}$ is less correlated to the output than ASA (Table 22. Consequently, $\beta_{R \mid I_{A}}^{\mathrm{ANN}}$, which equals 0.326 , is larger than $\beta_{R \mid A S A}^{\mathrm{ANN}}(0.079)$, whereas $\sigma_{\mathrm{ANN}}(0.094)$ stays the same. Considering Eq. 15, the impact of $\sigma_{\mathrm{ANN}}$ is less evident on the $I_{A}$ curves than the ASA curves.

These analyses show the importance of the consideration of $\sigma_{\mathrm{ANN}}$ in the computation of the fragility curves, where DM results are provided by ANN simulations. Otherwise, the uncertainty in the fragility curves will be underestimated.

Besides, $\sigma_{\mathrm{ANN}, \text { stat }}$ of the test data is also computed. Among the 20 test data, the positions of the first four data with the highest $\sigma_{\mathrm{ANN} \text {,stat }}$ values are visualized in the input space in Figure 15 . The numbers in the

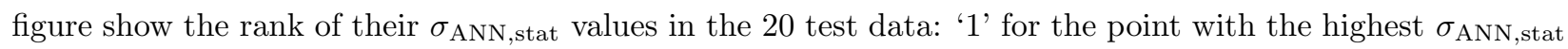
value, '2' for the second highest, etc. It can be observed that the test data with high values of $\sigma_{\mathrm{ANN} \text {,stat }}$ are located either at the lower boundary of the training data, or at the places where the training data are sparse, which is coherent with the property of $\sigma_{\mathrm{ANN}, \text { stat }}$ shown in Figure 5 .

\subsection{Fragility Curves}

After being trained, the ANN can be used to carry out fast-running simulations. For this purpose, a large number of seismic IMs have to be generated to represent the seismic motions. In this paper, the following statistical properties of the log-normal distributions of ASA and $I_{A}$ are obtained from the 100 triplets of seismic signals on the free surface (Table 5). The assumption of log-normality of the selected IMs has been validated in Section 4.2. With the large number of simulation results provided by the ANN, both methods 
presented in Section 3.5 can be applied for the computation of fragility curves.

Table 5: Statistics of ASA and $I_{A}$ on the free surface

\begin{tabular}{cccc}
\hline IM & Median & Log. standard deviation & $\rho\left(\right.$ ASA- $\left.I_{A}\right)$ \\
\hline ASA $[\mathrm{g}]$ & 2.28 & 0.417 & 0.846 \\
$I_{A}[\mathrm{~m} / \mathrm{s}]$ & 13.13 & 0.842 & \\
\hline
\end{tabular}

For the log-normal based fragility curve, 10,000 ASA- $I_{A}$ samples are generated with the statistics in Table 5. 10,000 ANN simulations are performed with these generated IMs, and the conditional probability of failure is computed with Eq. 15 . The computed fragility curve is described by ASA with median capacity $3.32 g$ and uncertainty $\beta_{\text {Total }}=0.127$, including $\sigma_{\mathrm{ANN}}=0.094$.

The pointwise fragility analysis is performed by conditional sampling of $I_{A}$ for a given value of ASA, since a conditional bivariate normal distribution is also normally distributed. In the analysis, the values of ASA are selected in $[2.2 g, 4.4 g]$ with $\Delta \mathrm{ASA}=0.1 g$. For every ASA value, $10,000 I_{A}$ are generated. At every ASA, the probability of failure is computed from Eq. 16, and the CIs are determined with Eq. 17. Fragility curves computed with both methods are shown in Figure 16.

Regarding the fragility curves, although there exist some differences between the log-normal based fragility curve and the $\mathrm{MC}$ estimation, the log-normal curve stays coherent with the pointwise MC curve. The lognormal assumption can be thus confirmed in this study. It is recalled that the source of the confidence intervals comes only from the paucity of the training data of the ANN.

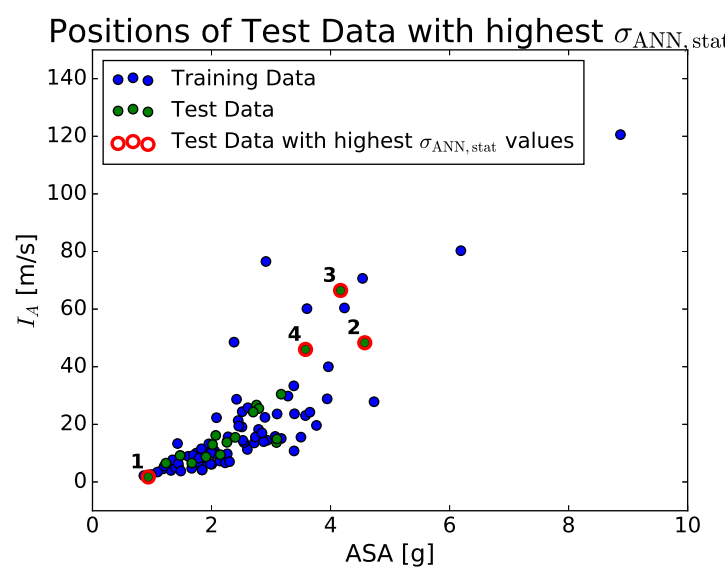

Figure 15: Positions of test data with highest $\sigma_{\mathrm{ANN}}$,stat

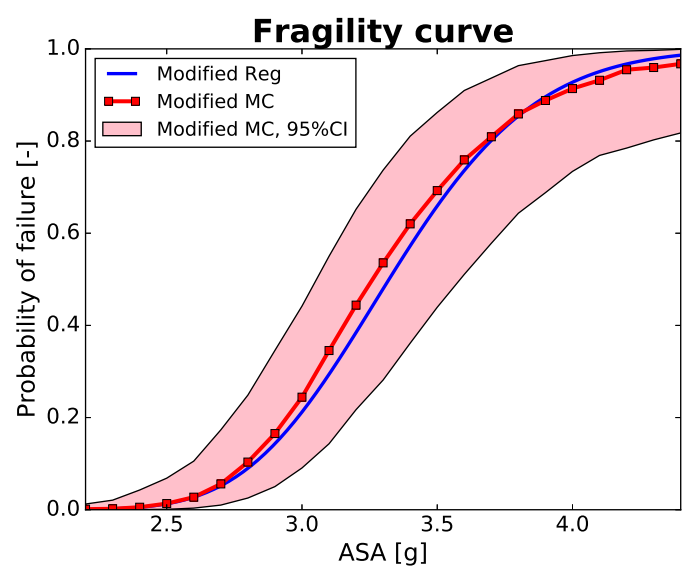

Figure 16: Fragility curves with ANN

\subsection{Discussions}

The proposed ANN-based fragility analysis has been applied to the K-K NPP to compute the fragility curve of an electrical cabinet. The assumptions made in this methodology are discussed what follows. The computational cost of the FEM analyses is also provided.

1. This study only considers seismic randomness in the FEM simulation. Uncertainties on structural parameters are not modeled. With other source of uncertainties, the seismic IM is less correlated to the structural output, so that the influence of the aleatory component of the metamodel uncertainty is less evident. 
2. Moreover, the variability in the responses of the considered electrical cabinet is dominated by the seismic record-to-record randomness. To justify this, the material uncertainties of concrete in Table 6] is considered for the first 50 seismic excitations. The values of the coefficients of variation of Table 6 are selected according to our expertise. The log-normal model of the material parameters has been suggested and used in [3, 52, 24, 15, 27]. Additionally, the log-normal distribution can ensure that all the values of material parameters are positive, in particular for small value parameters, such as the Poisson's ratio and the modal damping ratio. 50 FEM simulations are performed with the material uncertainties, with the stochastic values of Table 6 applied to the entire structure. The material parameters do not vary for elements within the structure. The corresponding fragility curve is computed with Reg method. Meanwhile, a fragility curve is calculated also with Reg method based on the first 50 FEM simulation results of this study (i.e. without the consideration of material uncertainties). Seismic excitations for both cases remain the same. The comparison of two fragility curves is shown in Figure 17 . It can be clearly observed that there is no obvious difference between the two fragility curves, which implies that the impact of the material randomness can be neglected compared to the earthquake randomness. That is another reason why material uncertainties are not modeled in this study. With uncertainty uniquely from seismic ground motions, the selection of IMs becomes therefore crucial to ensure the accuracy of the ANN.

Table 6: Uncertainties in material parameters of concrete in K-K NPP

\begin{tabular}{cccc}
\hline Material parameters & Distribution & Median & Coefficient of variation \\
\hline Young's modulus & Log-normal & $31,300 \mathrm{MPa}$ & 0.2 \\
Poisson ratio & Log-normal & 0.2 & 0.1 \\
Density & Log-normal & $2500 \mathrm{~kg} / \mathrm{m}^{3}$ & 0.05 \\
Modal damping ratio & Log-normal & 0.05 & 0.4 \\
\hline
\end{tabular}

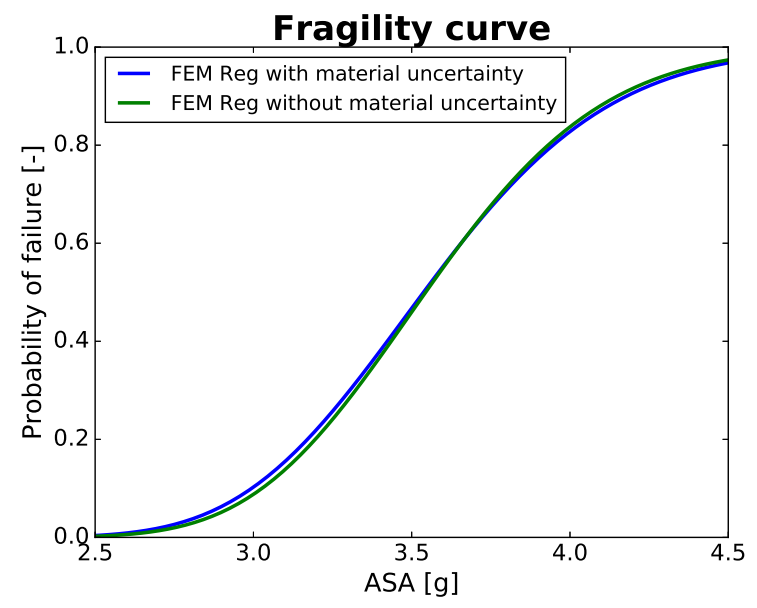

Figure 17: Comparison between fragility curves with or without material uncertainties

3. The ANN training errors are assumed to follow a normal distribution. The validity of this assumption is confirmed in this study. In addition, with a more mathematically rigorous derivation, the ANN prediction uncertainty should have followed a student $\mathrm{t}$ distribution. This approximation by a normal distribution allows the decomposition of the ANN prediction uncertainty into two normal aleatory and 
epistemic components.

4. The computational cost of the FEM simulations is listed in Table 7 Based on the soil impedances computed by BEM, one single FEM analysis takes $(120.02+66.86) / 100=1.87$ hours on an Intel Xeon E5-2600V2 CPU of 2.7GHz, which makes it almost unaffordable to run a large number of FEM simulations for the pointwise MC fragility analysis. However, once the ANN metamodel is established, the pointwise MC fragility analysis can be conducted within 0.25 hours. It has to be noticed that the ANN metamodel is constructed from the results of 100 FEM simulations, which means that $132.41+$ $120.02+66.86=319.29$ hours of mechanical simulations are the prerequisites for the ANN metamodel construction.

Table 7: Computational cost for numerical analyses

\begin{tabular}{ccc}
\hline Type of analyses & Number of analyses & Total computational time \\
\hline BEM for soil impedances & 4 & 132.41 hours \\
ELM analyses & 100 & 120.02 hours \\
SSI analyses & 100 & 66.86 hours \\
ANN simulations for MC fragility analysis & $10,000 \times 23$ & 0.25 hours \\
\hline
\end{tabular}

5. The applicability of the methodology is not limited to the K-K NPP case study. It can be generalized to other structures, by adopting a proper FEM analysis for the structures in question. The same procedure from Section 3.2 to Section 3.5 can be applied to conduct the fragility analysis. However, one should pay attention to the log-normal hypothesis made in the feature selection (Section 3.2) and generation of IMs (Section 3.5. If the log-normal assumption were not valid, an additional Nataf transformation [39] of the probability distribution would have to be performed, to transform arbitrary RVs to Gaussian RVs. It is also worth emphasizing that this methodology is not restricted to ANNs: the filter feature selection is independent of the metamodel; the delta method can also be applied to other models (e.g. polynomial response surfaces) to evaluate the metamodel uncertainty. The only difference is the way to compute the gradient.

\section{Summary \& Conclusions}

A methodology of ANN metamodels for the computation of fragility curves has been proposed in this paper. The ANN metamodel is utilized to build the statistical relation between the seismic intensity measures and the structural response. Once trained, the ANN metamodel allows carrying out a large number of simulations for both parametric and non-parametric fragility analyses, at negligible computational cost. Based on FEM simulation results, this methodology mainly consists of:

- Selection of the most relevant seismic intensity measure features. A filter approach based on semi-partial correlation coefficients is proposed in this study. It is compared with a wrapper approach based on GA. In the case study considered, the filter selection method shows slightly more advantages, in terms of accuracy and efficiency. Once the features have been retained, the ANN is trained with early stopping to prevent overfitting.

- Identification of the aleatory uncertainty and the epistemic uncertainty components in the ANN prediction uncertainties. The aleatory uncertainty is incorporated in the fragility curve, while the epistemic 
uncertainty is used to compute the confidence intervals.

- Computation of fragility curves and their confidence intervals, with both log-normal assumption and pointwise MC methods. The Reg and MC methods are adapted to take into account the ANN prediction uncertainties. The non-parametric MC fragility curve is used to verify the log-normal assumption, which is widely adopted in the fragility analysis.

Instead of methods based on repeated ANN trainings realized in [31, more efficient algorithms for the feature selection and the ANN prediction uncertainty quantification have been applied. Moreover, the ANN prediction uncertainty has been discussed thoroughly. This methodology has been applied to an industrial complex case study, i.e. Kashiwazaki-Kariwa nuclear power plant in Japan to evaluate the robustness of an electrical cabinet. The fragility curve computed with the log-normal assumption is described by ASA with median capacity $3.32 g$ and uncertainty $\beta_{\text {Total }}=0.127$. In addition, compared to the results of the pointwise MC estimation in this study, it is reasonable to assume a log-normal distribution for the fragility curves.

\section{Acknowledgement}

The authors want to thank the two anonymous reviewers for their valuable comments to this work. The contribution of this work is incorporated in the project NARSIS H2020 (New Approach to Reactor Safety ImprovmentS, Horizon 2020).

\section{References}

[1] EPRI, Seismic probabilistic risk assessment implementation guide, Tech. rep., Electric Power Research Institute EPRI, Palo Alto, CA, report 1002989 (2013).

[2] EPRI, Methodology for developing seismic fragilities, Tech. rep., Electric Power Research Institute EPRI, Palo Alto, CA, report TR-103959 (1994).

[3] R. Kennedy, C. Cornell, R. Campell, S. Kaplan, H. Perla, Probabilistic seismic safety study of an existing nuclear power plant, Nucl. Eng. Des. 59 (1980) 315-338. doi:10.1016/0029-5493(80)90203-4.

[4] C. Mai, K. Konakli, B. Sudret, Seismic fragility curves for structures using non-parametric representations, Front. Struct. Civ. Eng. 11 (2017) 169186. doi:10.1007/s11709-017-0385-y.

[5] I. Zentner, M. Gündel, N. Bonfils, Fragility analysis methods: Review of existing approaches and application, Nucl. Eng. Des.In press. doi:10.1016/j.nucengdes.2016.12.021.

[6] H. Y. Noh, D. Lallemant, A. S. Kiremidjian, Development of empirical and analytical fragility functions using kernel smoothing methods, Earthquake Eng. Struct. Dyn. 44 (2015) 1163-1180. doi:10.1002/ eqe.2505.

[7] D. Lallemant, A. Kiremidjian, H. Burton, Statistical procedures for developing earthquake damage fragility curves, Earthquake Eng. Struct. Dyn. 44 (2015) 13731389. doi:10.1002/eqe.2522.

[8] M. Shinozuka, M. Q. Feng, J. Lee, T. Naganuma, Statistical analysis of fragility curves, J. Eng. Mech. 126 (2000) 1224-1231. doi:10.1061/(ASCE)0733-9399(2000)126:12(1224). 
[9] I. Gidaris, A. A. Taflanidis, G. P. Mavroeidis, Kriging metamodeling in seismic risk assessment based on stochastic ground motion models, Earthquake Eng. Struct. Dyn. 44 (2015) 2377-2399. doi:10.1002/ eqe. 2586 .

[10] C. A. Cornell, F. Jalayer, R. O. Hamburger, D. A. Foutch, Probabilistic basis for 2000 SAC federal emergency management agency steel moment frame guidelines, J. Struct. Eng. 128 (2002) 526-533. doi : 10.1061/(ASCE) 0733-9445(2002)128:4(526).

[11] M. Perrault, Evaluation de la vulnérabilité sismique de bâtiments à partir de mesures in situ, Ph.D. thesis, Université de Grenoble (2013).

[12] H. Xu, P. Gardoni, Probabilistic capacity and seismic demand models and fragility estimates for reinforced concrete buildings based on three-dimensional analyses, Eng. Struct. 112 (2016) 200-214. doi:10.1016/j.engstruct.2016.01.005.

[13] I. Zentner, E. Borgonovo, Construction of variance-based metamodels for probabilistic seismic analysis and fragility assessment, Georisk 8 (2014) 202-216. doi:10.1080/17499518.2014.958173.

[14] V. U. Unnikrishnan, A. M. Prasad, B. N. Rao, Development of fragility curves using high-dimensional model representation, Earthquake Eng. Struct. Dyn. 42 (2013) 419-430. doi:10.1002/eqe.2214.

[15] N. Buratti, B. Ferracuti, M. Savoia, Response surface with random factors for seismic fragility of reinforced concrete frames, Struct. Saf. 32 (2010) 42-51. doi:10.1016/j.strusafe.2009.06.003

[16] J. Seo, D. G. Linzell, Use of response surface metamodels to generate system level fragilities for existing curved steel bridges, Eng. Struct. 52 (2013) 642-653. doi:10.1016/j.engstruct.2013.03.023.

[17] J. Seo, L. Dueas-Osorio, J. I. Craig, B. J. Goodno, Metamodel-based regional vulnerability estimate of irregular steel moment-frame structures subjected to earthquake events, Eng. Struct. 45 (2012) 585-597. doi:10.1016/j.engstruct.2012.07.003.

[18] S. K. Saha, V. Matsagar, S. Chakraborty, Uncertainty quantification and seismic fragility of baseisolated liquid storage tanks using response surface models, Probab. Eng. Mech. 43 (2016) 20-35. doi: $10.1016 / j \cdot$ probengmech.2015.10.008

[19] J. Park, P. Towashiraporn, Rapid seismic damage assessment of railway bridges using the responsesurface statistical model, Struct. Saf. 47 (2014) 1-12. doi:10.1016/j.strusafe.2013.10.001.

[20] N. D. Lagaros, M. Fragiadakis, Fragility assessment of steel frames using neural networks, Earthquake Spectra 23 (2007) 735-752. doi:10.1193/1.2798241.

[21] N. D. Lagaros, Y. Tsompanakis, P. N. Psarropoulos, E. C. Georgopoulos, Computationally efficient seismic fragility analysis of geostructures, Comput. Struct. 87 (2009) 1195-1203. doi:10.1016/j. compstruc.2008.12.001.

[22] C. C. Mitropoulou, M. Papadrakakis, Developing fragility curves based on neural network IDA predictions, Eng. Struct. 33 (2011) 3409-3421. doi:10.1016/j.engstruct.2011.07.005 
[23] E. Ferrario, N. Pedroni, E. Zio, F. Lopez-Caballero, Application of metamodel-based techniques for the efficient seismic analysis of structural systems, in: Safety and Reliability of Complex Engineered Systems, ESREL, 2015, pp. 1193-1200.

[24] A. Calabresea, C. G. Lai, Fragility functions of blockwork wharves using artificial neural networks, Soil Dyn. Earthquake Eng. 52 (2013) 88-102. doi:10.1016/j.soildyn.2013.05.002.

[25] S. Mangalathu, J.-S. Jeon, R. DesRoches, Critical uncertainty parameters influencing seismic performance of bridges using lasso regression, Earthquake Eng. Struct. Dyn doi:10.1002/eqe.2991.

[26] P. Gehl, D. D'Ayala, Development of bayesian networks for the multi-hazard fragility assessment of bridge systems, Struct. Saf. 60 (2016) 3746. doi:10.1016/j.strusafe.2016.01.006.

[27] J. Ghosh, J. E. Padgett, L. Dueas-Osorio, Surrogate modeling and failure surface visualization for ef ficient seismic vulnerability assessment of highway bridges, Probab. Eng. Mech. 34 (2013) 189-199. doi:10.1016/j.probengmech.2013.09.003

[28] G. Jia, A. A.Taflanidis, Kriging metamodeling for approximation of high-dimensional wave and surge responses in real-time storm/hurricane risk assessment, Comput. Methods in Appl. Mech. Eng. 261-262 (2013) 24-38. doi:10.1016/j.cma.2013.03.012.

[29] C. V. Mai, M. D. Spiridonakos, E. N. Chatzi, B. Sudret, Surrogate modelling for stochastic dynamical systems by combining narx models and polynomial chaos expansions, International Journal for Uncertainty Quantification 6 (2016) 419-430. doi:10.1615/Int.J.UncertaintyQuantification.v6.i4.

[30] N. Ataei, J. E. Padgett, Fragility surrogate models for coastal bridges in hurricane prone zones, Eng. Struct. 103 (2015) 203-213. doi:10.1016/j.engstruct.2015.07.002.

[31] E. Ferrario, N. Pedroni, E. Zio, F. Lopez-Caballero, Bootstrapped artificial neural networks for the seismic analysis of structural systems, Struct. Saf. 67 (2017) 70-84. doi:10.1016/j.strusafe.2017. 03.003 .

[32] P. Towashiraporn, Building seismic fragilities using response surface metamodels, Ph.D. thesis, Georgia Institute of Technology (2004).

[33] C. M. Bishop, Neural Networks for Pattern Recognition, Oxford University Press, 1995.

[34] R. D. Reed, R. J. Marks, Neural Smithing, MIT Press, 1999.

[35] IAEA, Review of seismic evaluation methodologies for nuclear power plants based on a benchmark exercise, Tech. rep., International Atomic Energy Agency (2013).

[36] B. R. Ellingwood, K. Kinali, Quantifying and communicating uncertainty in seismic risk assessment, Struct. Saf. 31 (2009) 179-187. doi:10.1016/j.strusafe.2008.06.001

[37] N. Yoshida, S. Kobayashi, I. Suetomi, K. Miura, Equivalent linear method considering frequency dependent characteristics of stiffness and damping, Soil. Dyn. Earthq. Eng. 22 (2002) 205-222. doi: 10.1016/S0267-7261(02)00011-8. 
[38] R. Kohavi, G. H. John, Wrappers for feature subset selection, Artificial Intelligence 97 (1997) $273-324$. doi : 10.1016/S0004-3702(97)00043-X

[39] O. Ditlevesen, H. Madsen, Structural Reliability Methods, 2005.

[40] D. Rumelhart, G. Hinton, R. Williams, Learning Internal Representations by Error Propagation in Parallel Distributed Processing: Explorations in the Microstructure of Cognition, The MIT Press, 1986.

[41] N. Pedroni, E. Zio, G. Apostolakis, Comparison of bootstrapped artificial neural networks and quadratic response surfaces for the estimation of the functional failure probability of a thermalhydraulic passive system, Reliab. Eng. Syst. Safe. 95 (2010) 386-395. doi:10.1016/j.ress.2009.11.009.

[42] E. Zio, A study of the bootstrap method for estimating the accuracy of artificial neural networks in predicting nuclear transient processes, IEEE T. Nucl. Sci. 53 (2006) 1460-1478. doi:10.1109/TNS. 2006.871662

[43] I. Rivals, L. Personnaz, Construction of confidence intervals for neural networks based on least squares estimation, Neural Networks 13 (2000) 463-484. doi:10.1016/S0893-6080(99)00080-5.

[44] G. Chryssoloiuris, M. Lee, A. Ramsey, Confidence interval prediction for neural network models, IEEE T. Neural Networ. 7 (1996) 229-232. doi:10.1109/72.478409.

[45] R. Dybowski, S. J. Roberts, Confidence intervals and prediction intervals for feed-forward neural networks, in: R. Dybowski, V. Gant (Eds.), Clinical Applications of Artificial Neural Networks, Cambridge University Press, 2001, pp. 298-326. doi:10.1016/S0933-3657(02)00081-7.

[46] K. W. Campbell, Y. Bozorgnia, NGA ground motion model for the geometric mean horizontal component of PGA, PGV, PGD and 5\% damped linear elastic response spectra for periods ranging from 0.01 to 10 s, Earthquake Spectra 24 (2008) 139-171. doi:10.1193/1.2857546.

[47] J. W. Baker, Correlation of ground motion intensity parameters used for predicting structural and geotechnical response, in: Proceedings of the 10th International Conference on Applications of Statistics and Probability in Civil Engineering, Tokyo, 2007.

[48] Code_Aster, opensource Finite Element code, http://www.code-aster.org.

[49] MISS, a software in earthquake engineering and structural dynamics, http://www.mssmat.ecp.fr/miss.

[50] J. P. Stewart, S.-J. Chiou, J. D. Bray, R. W. Graves, P. G. Somerville, N. A. Abrahamson, Ground motion evaluation procedures for performance-based design, Tech. rep., Pacific Earthquake Engineering Research Center (2001).

[51] M. D. Biasio, S. Grange, F. Dufour, F. Allain, I. Petre-Lazar, Intensity measures for probabilistic assessment of non-structural components acceleration demand, Earthquake Eng. Struct. Dyn. 44 (2015) 2261-2280. doi:10.1002/eqe.2582. 
[52] A. B. Liel, C. B. Haselton, G. G. Deierlein, J. W. Baker, Incorporating modeling uncertainties in the assessment of seismic collapse risk of buildings, Struct. Saf. 31 (2009) 197-211. doi:10.1016/j. strusafe.2008.06.002 